\title{
Miocene fern spores and pollen grains from the Solimões Basin, Amazon Region, Brazil
}

\section{Natália de Paula Sá1"and Marcelo de Araujo Carvalho'}

Received: April 27, 2017

Accepted: July 11, 2017

\begin{abstract}
This work documents fern spores and pollen grains (miospores) recovered from rocks of the Solimões Formation (Solimões Basin), their botanical affinities, ecology and distribution in the Miocene of the Amazon Region. The assemblage of miospores is well preserved and diverse. They are identified, illustrated and assigned to the ten families of ferns and 22 families of spermatophytes. All miospores were identified to the taxonomic level of species except for two taxa (Perinomonoletes and Podocarpidites). The families Pteridaceae and Arecaceae were most representative of ferns and spermatophytes, respectively. This work contributes to the knowledge of the paleoflora and will aid in paleoenvironmental, paleoecological and biostratigraphic interpretations of the Miocene of the Amazon Region.
\end{abstract}

Keywords: Miocene, miospores, Neogene, palynology, Solimões, vegetation

\section{Introduction}

The Amazon is the largest tropical rainforest ecosystem and its high diversity can be explained by ecological, environmental and paleontological models. Climate changes in the Pleistocene caused the expansion and retraction of the rainforest cycles. The changes were considered the triggers for the speciation and accumulation of species (Haffer 1969) and this theory has long been the basis for interpreting the current diversity patterns. However, numerous studies (e.g., Gentry 1982; Frailey 1986; Hooghiemstra \& Hammen 1998; Monsch 1998; Jaramillo et al. 2006; Cozzuol 2006; Hoorn et al. 2010a) have shown that the diversification of biota is pre-Quaternary, reassembling to the last $60 \mathrm{Ma}$ (million years).

The Neogene of Amazon region shows a very diversified flora. The records of this flora are based mainly on fossil woods and palynomorphs (e.g., spores, pollen grains) and a large part of this material allows establishing botanical affinities. For Miocene age, the palynology has been the most used technique to understand the past of the Amazon, especially miospores, which inform about the diversity and richness of the paleoflora. Several studies (e.g. Lorente 1986; Hoorn 1993; 1994a; b; c; Hoorn et al. 1995; Silva-Caminha et al. 2010; Hoorn et al. 2010b; Silveira \& Souza 2015; 2016; Leite et al. 2016; D’Apolito 2016) show a paleoflora rich in pteridophytes (Anemiaceae, Cyatheaceae, Polypodiaceae, Pteridaceae) and gymnosperms (Araucariaceae and Podocarpaceae). Angiosperms are a separate case because it is currently the most important flora in the Amazon region. Since the Miocene, almost all the families (e.g. Annonaceae, Arecaceae, Asteraceae, Bombacaceae, Euphorbiaceae, Fabaceae, Malvaceae, Melastomataceae, Malpighiaceae, Sapotaceae) were already present in the region (Gentry 1982; Burnham \& Graham 1999; Jaramillo et al. 2010; Hoorn et al. 2010c; 2017).

\footnotetext{
${ }^{1}$ Laboratório de Paleoecologia Vegetal, Departamento de Geologia e Paleontologia, Museu Nacional, Universidade Federal do Rio de Janeiro, 20940-040, Rio de Janeiro, RJ, Brazil
}

* Corresponding author: napaulasa@gmail.com 
The recognition of the Miocene flora also allows paleoecological and paleoenvironmental inferences, as well as support the biostratigraphy framework the region (Lorente 1986; Hoorn 1993; 1994c; Silva-Caminha et al. 2010; Leandro 2012; Silveira \& Souza 2015; 2016; Leite et al. 2016).

Therefore, this work seeks to inventory fern spores and pollen grains found in the Miocene rocks (Solimões Formation), their affinities and ecology in order to facilitate taxonomic identification and to support paleoenvironmental, paleoecological and biostratigraphic studies of the Miocene in the Amazon.

\section{Materials and methods}

\section{Study area}

The Solimões Basin is located in the western portion of the Amazon, bordered to the west by the Iquitos Arch and to the east by the Purus Arch. It is Paleozoic intracratonic depression, covering about $950,000 \mathrm{~km}^{2}$ (Barata \& Caputo 2007) is between $2^{\circ}-8^{\circ} \mathrm{S} 62^{\circ}-72^{\circ} \mathrm{W}$ (Fig. 1). Internally, there is a north-south regional control characterized to distribute the sediments in the basin, called the Carauari Arch. This subdivides the depression into the Jurua sub-basin to the east and the Jandiatuba sub-basin to the west (WanderleyFilho et al. 2007, Fig. 1).

According to Eiras et al. (1994), the Solimões Basin covers six depositional sequences: ordovician, silurodevonian, devonian-carboniferous, carboniferous-permian, cretaceous and cenozoic. In the last sequence are recognizing the Solimões and Içá formations.

The Solimões Formation extends for about $500,000 \mathrm{~km}^{2}$, with sedimentary thickness ranging from 300 to $400 \mathrm{~m}$, and can reach up to $1000 \mathrm{~m}$ near the Iquitos Arch. It covers Acre and west Amazonas, as well as the territories of Peru (Marañon and Putumayo basins) and Colombia (Caquetá and Putumayo basins) (Maia et al. 1977; Hoorn et al. 2010a). The Solimões Formation rocks comprise shales, siltstones and sandy shales, clayey silts and medium to fine-grained sands, lignites, carbonaceous clays and limestones (Maia et al. 1977).

In relation to the paleoenvironments during the Miocene, several studies indicate a heterogeneous and dynamic environment composed of rivers, lakes, flood plains, mangroves and coastal plains. It is also found elements of transitional and / or marine environments such

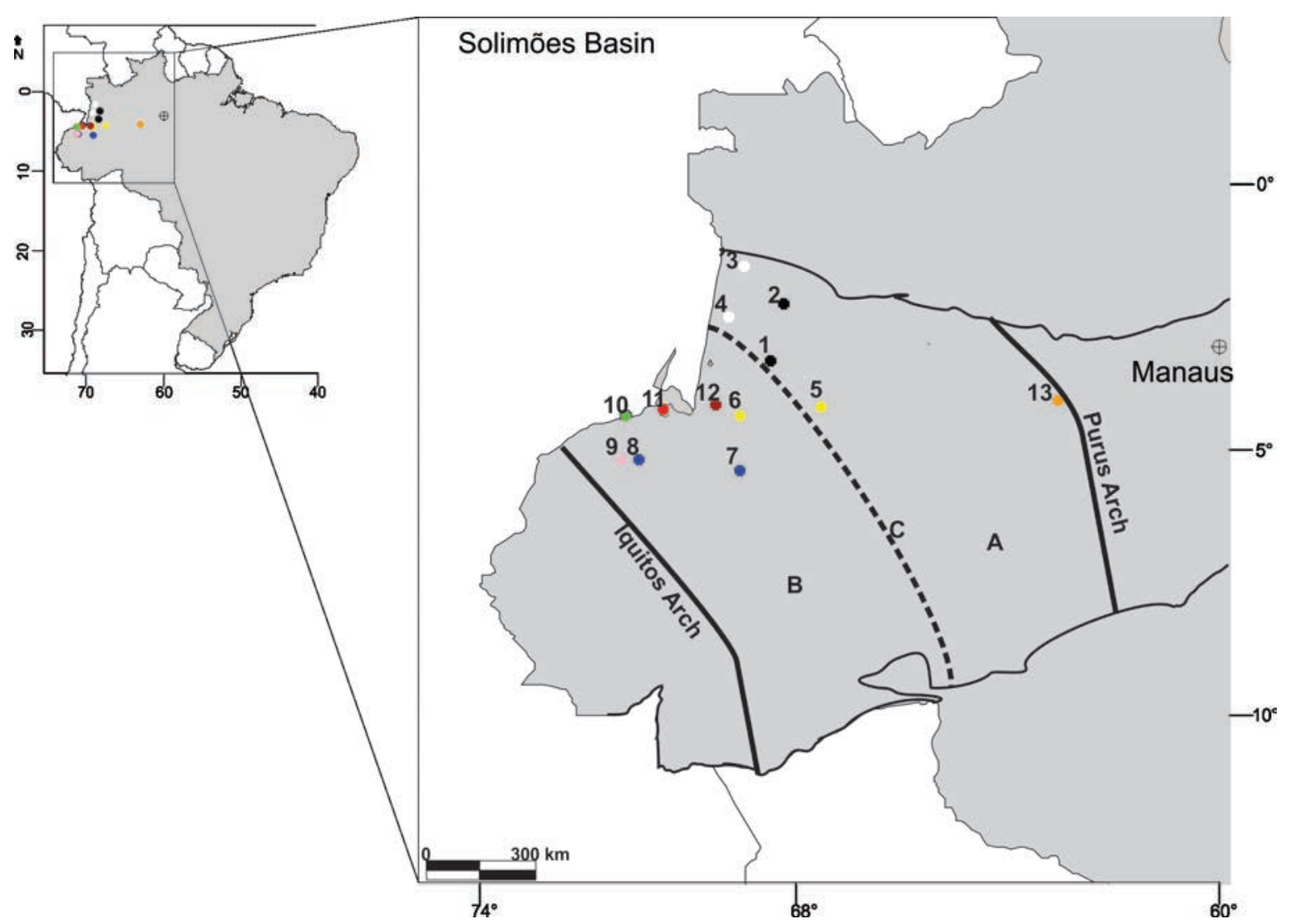

Figure 1. Solimões Basin location. A. Juruá sub-basin. B. Jandiatuba sub-basin. C. Carauari High. Legends: black dots 1-AS-37-AM (1) e 1-AS-46-AM (2); red dot: 1-AS-4a-AM (11); green dot: 1-AS-32-AM (10); yellow dots: 1-AS-19-AM (6) and 1-AS-27-AM (5); white dots: 1-AS-51-AM (4) and 1-AS-52-AM (3); blue dots: 1-AS-31-AM (7) and 1-AS-34-AM (8); pink dot: 1-AS-33-AM (9); brown dot: 1-AS-105-AM (12) and orange dot: Coari and Alto Solimões outcrops (13). Please see the PDF version for color reference. 
as microforaminifera linings, molluscs and dinoflagellate cysts (e.g. Hoorn 1993; 1994a; Räsänen et al. 1995; Latrubesse et al. 1997; 2007; 2010; Lovejoy 1998; Vonhof et al. 1998; 2003; Wesselingh et al. 2002; 2006; Wesselingh 2006; Wesselingh \& Salo 2006; Ramos 2006; Hoorn et al. 2010a; Gross et al. 2011; 2013; Linhares et al. 2011; Nogueira et al. 2013; Boonstra et al. 2015).

\section{Metodology}

In Solimões Basin investigations for energy resources were conducted by the federal government in the 1970s by the Geological Survey of Brazil (CPRM) and the National Department of Mineral Production (DNPM). The project entitled "Coal Alto Solimões" did a survey of areas with coal mining potential. A total of 84 wells was drilled in an area of $320,000 \mathrm{~km}^{2}$ in northwest Brazil and this material was deposited at CPRM/DNPM - Manaus - AM (Maia et al. 1977). The wells 1-AS-37-AM and 1-AS-46-AM were chosen for palynological analysis considering its position in the Solimões Basin and the state of conservation.

The well 1-AS-37-AM is at an altimetric elevation of $60 \mathrm{~m}$ and coordinates $03^{\circ} 30^{\prime} \mathrm{S} 68^{\circ} 51^{\prime} \mathrm{W}$ near the Jandiatuba River. Its thickness is $242.60 \mathrm{~m}$, and the initial $12 \mathrm{~m}$ corresponds to Holocene deposits in contact with the top of the Solimões Formation. The lithology is predominantly pelitic with higher occurrence of lignite layers (Maia et al. 1977).

The well 1-AS-46-AM is located in the northwestern portion of the Solimões Basin, coordinates $02^{\circ} 23^{\prime} S 68^{\circ} 28^{\prime} \mathrm{W}$, altimetry $101 \mathrm{~m}$ and thickness of $200.90 \mathrm{~m}$. In this well, the Içá Formation represents the initial $6.0 \mathrm{~m}$ in erosive contact with the Solimões Formation. The lithology is pelitic and there are fewer layers of lignite (Maia et al. 1977).

For the study were selected 100 core samples from each well, from which $10 \mathrm{~g} /$ sample was processed by standard technique in palynology to eliminate the inorganic material by means of acidic attacks (see Uesugui 1979; Erdtman 1969; Faegri \& Iversen 1966).

The miospores recovered from the cores were identified by comparison with works of Germeraad et al. (1968); Regali et al. (1974a; b); Lorente (1986); Hoorn (1993; 1994c); Silva-Caminha et al. (2010); Jaramillo et al. (2011); D’Apolito (2016) and the website http://biogeodb.stri.si.edu/jaramillo/ palynomorph/pollen, which hosts an atlas with images of several publications of palynomorphs from North of South America. The botanical affinities, ecology and distribution of spores and pollen grains were attributed according to the studies above and Tryon \& Lugardon (1991); Jaramillo et al. (2010; 2011); Jaramillo \& Rueda (2013) and Silveira \& Souza (2015; 2016).

The photographs were obtained in Axioplan microscope and AxioCam MRc camera, with 1000x magnification by the program Axiovision and processed in the software Corel Draw 17.0. Each miospore was referenced using the "England Finder" coordinates associated with the number slides. The slides were deposited in the Laboratory of Plant Paleoecology of the Department of Geology and Paleontology of the National Museum of the Federal University of Rio de Janeiro.

\section{Results}

Sixty miospores were selected for this work, which include 19 fern spores and 41 pollen grains. The spores are distributed into 10 families, being the family Pteridaceae the most frequent. The pollen grains are distributed into 22 families (according to Cronquist 1988), with emphasis on the family Arecaceae. The miospores were systematized in two categories: spores and pollen grains, following the alphabetical order. Botanical affinity, ecology and distribution in the Solimões Formation (Tab. 1, Fig. 1) were attributed based on the literature.

Anteturma SPORITES Potonié 1893

Genus Cingulatisporites Thomson emend. Potonié 1956

Cingulatisporites laevigatus Silva-Caminha et al. 2010

(Fig. 2A)

Botanical affinity: unknown

Ecology: unknown

Genus Crassoretitriletes Germeraad et al, 1968

Crassoretitriletes vanraadshoovenii Germeraad et al. 1968

(Fig. 2B)

Ecology: pantropical, it occurs throughout South America (Tryon \& Lugardon 1991), wetlands and swamps.

Genus Deltoidospora Miner 1935

Deltoidospora adriennis (Potonié \& Gelletich 1933)

Fredericksen 1983 (Fig. 2C)

Botanical affinity: family Pteridaceae, Acrostichum aureum

Ecology: pantropical, it occurs in coastal environments on all continents (Tryon \& Lugardon 1991), mangrove (Jaramillo et al. 2010)

Genus Distaverrusporites Muller 1968

Distaverrusporites margaritatus Muller 1968 (Fig. 2D)

Botanical affinity: unknown

Ecology: unknown

Genus Echinatisporis Krutzsch 1959

Echinatisporis infantus D'Apolito 2016 (Fig. 2E)

Botanical affinity: families Thelypteraceae/Athyriaceae/ Marathiaceae

Ecology: unknown

Echinatisporis muelleri Krutzsch 1967 (Fig. 2F)

Botanical affinity: families Thelypteraceae/Athyriaceae/

Marathiaceae (D’Apolito 2016)

Ecology: unknown 
Table 1. Miospores documented in this study and in the previous works for Solimões Formation. For the location of the wells, see Figure 1.

\begin{tabular}{|c|c|c|c|c|c|c|c|c|c|}
\hline \multirow[b]{2}{*}{ Miospores } & \multicolumn{8}{|c|}{ Records in Solimões Formation* } & \multirow[b]{2}{*}{ No/EF } \\
\hline & 1-AS-04-AM & 1-AS-32-AM & $\begin{array}{l}\text { 1-AS-19-AM } \\
\text { 1-AS-27-AM }\end{array}$ & $\begin{array}{l}1-A S-51-A M \\
1-A S-52-A M\end{array}$ & $\begin{array}{l}\text { 1-AS-31-AM } \\
1-A S-34-A M\end{array}$ & 1-AS-33-AM & 1-AS-105-AM & $\begin{array}{c}\text { Coari e } \\
\text { Alto Solimões }\end{array}$ & \\
\hline Cingulatisporites laevigatus & & & $\mathrm{X}$ & & & & & & $\begin{array}{c}\text { 1-AS-46-AM } \\
655 / W 10\end{array}$ \\
\hline Crassoretitriletes vanraadshoovenii & $\mathrm{X}$ & $\mathrm{X}$ & $\mathrm{X}$ & $\mathrm{X}$ & $\mathrm{X}$ & $\mathrm{X}$ & $\mathrm{X}$ & $\mathrm{X}$ & $\begin{array}{c}\text { 1-AS-37-AM 379/ } \\
\text { W60 }\end{array}$ \\
\hline Deltoidospora adriennis & $\mathrm{X}$ & $\mathrm{X}$ & $\mathrm{X}$ & $\mathrm{X}$ & & & $\mathrm{X}$ & $\mathrm{X}$ & $\begin{array}{c}\text { 1-AS-46-AM } \\
\text { 588/W51-2 }\end{array}$ \\
\hline Distaverrusporites margaritatus & & & $\mathrm{X}$ & $\mathrm{X}$ & & & $\mathrm{X}$ & & $\begin{array}{c}\text { 1-AS-46-AM 588/ } \\
\text { X64-4 }\end{array}$ \\
\hline Echinatisporis infantus & & & & & & & $\mathrm{X}$ & & $\begin{array}{c}\text { 1-AS-46-AM } \\
698 / 056-4\end{array}$ \\
\hline Echinatisporis muelleri & & & $\mathrm{X}$ & & $\mathrm{X}$ & & $\mathrm{X}$ & $\mathrm{X}$ & $\begin{array}{c}\text { 1-AS-46-AM 579/ } \\
\text { Z60-4 }\end{array}$ \\
\hline Kuylisporites waterbolkii & & & $\mathrm{X}$ & & & & $\mathrm{X}$ & $\mathrm{X}$ & $\begin{array}{c}\text { 1-AS-46-AM } \\
670 / Y 64-2\end{array}$ \\
\hline Laevigatosporites tibuiensis & & & $\mathrm{X}$ & & & & $\mathrm{X}$ & & $\begin{array}{c}\text { 1-AS-37-AM } \\
\text { 577/Q68 }\end{array}$ \\
\hline Magnastriatites grandiosus & $\mathrm{X}$ & $\mathrm{X}$ & $\mathrm{X}$ & $\mathrm{X}$ & & $\mathrm{X}$ & $\mathrm{X}$ & $\mathrm{X}$ & $\begin{array}{l}1-A S-37-A M \\
385-2 / O 57-4\end{array}$ \\
\hline Nijssenosporites fossulatus & & & & & & $\mathrm{X}$ & $\mathrm{X}$ & & $\begin{array}{c}\text { 1-AS-37-AM } \\
\text { 373/W63-4 }\end{array}$ \\
\hline Perinomonoletes $s p$. & & & & & & & & $\mathrm{X}$ & $\begin{array}{c}\text { 1-AS-46-AM } \\
693 / \mathrm{X} 53-4\end{array}$ \\
\hline Polypodiaceoisporites amazonensis & & & $\mathrm{X}$ & & & & & & $\begin{array}{c}\text { 1-AS-37-AM } \\
\text { 398/Y35-1 }\end{array}$ \\
\hline Polypodiaceoisporites potoniei & $\mathrm{X}$ & & & $\mathrm{X}$ & & & & $\mathrm{X}$ & $\begin{array}{c}1-\mathrm{AS}-46-\mathrm{AM} \\
643 / \mathrm{Y} 60-3\end{array}$ \\
\hline Polypodiisporites aff. specious & & & $\mathrm{X}$ & & & & $\mathrm{X}$ & & $\begin{array}{c}\text { 1-AS-46-AM } \\
695 / R 66-1\end{array}$ \\
\hline Psilatriletes lobatus & & & $\mathrm{X}$ & & & & $\mathrm{X}$ & & $\begin{array}{l}\text { 1-AS-37-AM } \\
573 / Z 29-03\end{array}$ \\
\hline Retitriletes sommeri & & & & & & & & & $\begin{array}{c}\text { 1-AS-46-AM } \\
755 / S 57-1\end{array}$ \\
\hline Verrucatosporites usmensis & & $\mathrm{X}$ & & $\mathrm{X}$ & & & & $\mathrm{X}$ & $\begin{array}{l}\text { 1-AS-37-AM } \\
\text { 573/N53-2-4 }\end{array}$ \\
\hline Verrucatotriletes bullatus & $\mathrm{X}$ & & $\mathrm{X}$ & & & & & $\mathrm{X}$ & $\begin{array}{c}\text { 1-AS-46-AM } \\
696 / \text { K74 }\end{array}$ \\
\hline Verrucatotriletes etayoi & & & $\mathrm{X}$ & $\mathrm{X}$ & & & & & $\begin{array}{c}\text { 1-AS-46-AM } \\
746 / \mathrm{X} 69-4\end{array}$ \\
\hline Cyclusphaera scabrata & & & & & & & $\mathrm{X}$ & & $\begin{array}{c}\text { 1-AS-37-AM } \\
\text { 478/W62-4 }\end{array}$ \\
\hline Podocarpidites sp. & $\mathrm{X}$ & $\mathrm{X}$ & $\mathrm{X}$ & $\mathrm{X}$ & & $\mathrm{X}$ & & $\mathrm{X}$ & $\begin{array}{c}\text { 1-AS-46-AM } \\
\text { 703/U52-3 }\end{array}$ \\
\hline Arecipites perfectus & & & $\mathrm{X}$ & $\mathrm{X}$ & & & $\mathrm{X}$ & & $\begin{array}{c}\text { 1-AS-37-AM } \\
\text { 535/R75-3 }\end{array}$ \\
\hline Bombacacidites baculatus & $\mathrm{X}$ & & $\mathrm{X}$ & $\mathrm{X}$ & $\mathrm{X}$ & $\mathrm{X}$ & $\mathrm{X}$ & $\mathrm{X}$ & $\begin{array}{c}\text { 1-AS-37-AM } \\
\text { 513/V59-1 }\end{array}$ \\
\hline Bombacacidites fossulatus & & $\mathrm{X}$ & $\mathrm{X}$ & $\mathrm{X}$ & $\mathrm{X}$ & & & & $\begin{array}{c}\text { 1-AS-37-AM } \\
379 / Z 62-1\end{array}$ \\
\hline Bombacacidites lorenteae & $\mathrm{X}$ & & & & & & $\mathrm{X}$ & & $\begin{array}{c}1-\mathrm{AS}-46-\mathrm{AM} \\
588 / \mathrm{S} 58-2\end{array}$ \\
\hline Bombacacidites nacimientoensis & $\mathrm{X}$ & $\mathrm{X}$ & $\mathrm{X}$ & $\mathrm{X}$ & & & $\mathrm{X}$ & $\mathrm{X}$ & $\begin{array}{c}1-\mathrm{AS}-37-\mathrm{AM} \\
535 / \mathrm{Y}-62\end{array}$ \\
\hline
\end{tabular}


Table 1. Cont.

\begin{tabular}{|c|c|c|c|c|c|c|c|c|c|}
\hline \multirow[b]{2}{*}{ Miospores } & \multicolumn{8}{|c|}{ Records in Solimões Formation* } & \multirow[b]{2}{*}{$\mathrm{No} / \mathrm{EF}$} \\
\hline & 1-AS-04-AM & 1-AS-32-AM & $\begin{array}{l}1-A S-19-A M \\
1-A S-27-A M\end{array}$ & $\begin{array}{l}\text { 1-AS-51-AM } \\
\text { 1-AS-52-AM }\end{array}$ & \begin{tabular}{|} 
1-AS-31-AM \\
1-AS-34-AM
\end{tabular} & 1-AS-33-AM & 1-AS-105-AM & $\begin{array}{l}\text { Coari e Alto } \\
\text { Solimões }\end{array}$ & \\
\hline Cichoreacidites longispinosus & & & $\mathrm{X}$ & & $\mathrm{X}$ & $\mathrm{x}$ & $\mathrm{X}$ & $\mathrm{X}$ & $\begin{array}{c}\text { 1-AS-37-AM } \\
\text { 378/T50 }\end{array}$ \\
\hline Corsinipollenites oculusnoctis & $\mathrm{X}$ & $\mathrm{X}$ & $\mathrm{X}$ & $\mathrm{X}$ & $\mathrm{X}$ & $\mathrm{X}$ & $\mathrm{X}$ & & $\begin{array}{c}\text { 1-AS-37-AM } \\
\text { 379/Y71 }\end{array}$ \\
\hline Echiperiporites akanthos & $\mathrm{X}$ & & $\mathrm{X}$ & & & $\mathrm{X}$ & $\mathrm{X}$ & $\mathrm{X}$ & $\begin{array}{l}\text { 1-AS-46-AM } \\
589 / Z 33-4\end{array}$ \\
\hline Echiperiporites estelae & $\mathrm{x}$ & $\mathrm{X}$ & $\mathrm{X}$ & $\mathrm{X}$ & & $\mathrm{X}$ & $\mathrm{X}$ & $\mathrm{X}$ & $\begin{array}{c}\text { 1-AS-37-AM } \\
\text { 573/L56 }\end{array}$ \\
\hline Echiperiporites lophatus & & & $\mathrm{X}$ & & & $\mathrm{X}$ & $\mathrm{X}$ & & $\begin{array}{l}\text { 1-AS-37-AM } \\
557 / \text { W24-3-4 }\end{array}$ \\
\hline Echiperiporites scrabrannulatus & & & & & & & & & $\begin{array}{c}\text { 1-AS-37-AM } \\
385 / \mathrm{P} 60\end{array}$ \\
\hline Echitricolporites spinosus & $\mathrm{X}$ & $\mathrm{X}$ & $\mathrm{X}$ & $\mathrm{X}$ & $\mathrm{X}$ & $\mathrm{x}$ & $\mathrm{X}$ & $\mathrm{X}$ & $\begin{array}{c}\text { 1-AS-46-AM } \\
\text { 535/R69-1 }\end{array}$ \\
\hline Echitriporites trianguliformis & & & $\mathrm{X}$ & & & & & & $\begin{array}{c}\text { 1-AS-46-AM } \\
695 / R 51-4\end{array}$ \\
\hline Fenestristes garciae & & & & $\mathrm{X}$ & & & & & $\begin{array}{c}\text { 1-AS-37-AM } \\
535 / \mathrm{Q} 61-3\end{array}$ \\
\hline Fenestrites spinosus & & & & $\mathrm{X}$ & $\mathrm{X}$ & $\mathrm{X}$ & $\mathrm{X}$ & $\mathrm{X}$ & $\begin{array}{c}\text { 1-AS-37-AM } \\
513 / \mathrm{N} 42-4\end{array}$ \\
\hline Grimsdalea magnaclavata & $\mathrm{X}$ & $\mathrm{X}$ & $\mathrm{X}$ & $\mathrm{X}$ & $\mathrm{X}$ & $\mathrm{X}$ & $\mathrm{X}$ & $\mathrm{X}$ & $\begin{array}{c}\text { 1-AS-46-AM } \\
\text { 746/W62-4 }\end{array}$ \\
\hline Inaperturopollenites solimoensis & & & $\mathrm{X}$ & & & $\mathrm{X}$ & & & $\begin{array}{c}\text { 1-AS-37-AM } \\
573 / J 57-1\end{array}$ \\
\hline Ladakhipollenites? caribbiensis & & & $\mathrm{x}$ & $\mathrm{X}$ & $\mathrm{X}$ & $\mathrm{x}$ & $\mathrm{X}$ & $\mathrm{X}$ & $\begin{array}{c}\text { 1-AS-46-AM } \\
\text { 740/K52 }\end{array}$ \\
\hline Loranthacites digitatus & & & $\mathrm{x}$ & & & & & & $\begin{array}{c}\text { 1-AS-37-AM } \\
483 / Y 43-4\end{array}$ \\
\hline Malvacipollis spinulosa & & & & $\mathrm{X}$ & & & $\mathrm{X}$ & & $\begin{array}{c}\text { 1-AS-37-AM } \\
\text { 561/M61-3-4 }\end{array}$ \\
\hline Malvacipolloides maristellae & $\mathrm{X}$ & $\mathrm{X}$ & $\mathrm{X}$ & $\mathrm{X}$ & $\mathrm{X}$ & & $\mathrm{X}$ & & $\begin{array}{c}\text { 1-AS-37-AM } \\
573 / \mathrm{U} 63-2\end{array}$ \\
\hline Margocolporites "hornii" & & & & & & & & & $\begin{array}{c}\text { 1-AS-37-AM } \\
\text { 411/W61-2 }\end{array}$ \\
\hline Margocolporites vanwijhei & $\mathrm{X}$ & & $\mathrm{X}$ & & $\mathrm{X}$ & $\mathrm{X}$ & $\mathrm{X}$ & & $\begin{array}{c}\text { 1-AS-37-AM } \\
533 / S 63-3\end{array}$ \\
\hline Mauritiidites franciscoi var. franciscoi & $\mathrm{X}$ & $\mathrm{X}$ & $\mathrm{X}$ & $\mathrm{X}$ & & $\mathrm{X}$ & $\mathrm{X}$ & $\mathrm{X}$ & $\begin{array}{c}\text { 1-AS-37-AM } \\
411 / S 63-1\end{array}$ \\
\hline Perisyncolporites pokornyi & $\mathrm{X}$ & $\mathrm{X}$ & $\mathrm{X}$ & $\mathrm{X}$ & & $\mathrm{X}$ & $\mathrm{X}$ & $\mathrm{X}$ & $\begin{array}{c}\text { 1-AS-46-AM } \\
\text { 588/W54 }\end{array}$ \\
\hline Polyadopollenites marileae & & & & & & $\mathrm{X}$ & & & $\begin{array}{c}\text { 1-AS-46-AM } \\
602 / Y 24\end{array}$ \\
\hline Proteacidites triangulatus & & & $\mathrm{X}$ & $\mathrm{X}$ & & $\mathrm{x}$ & $\mathrm{X}$ & $\mathrm{x}$ & $\begin{array}{c}\text { 1-AS-37-AM } \\
528 / \mathrm{Z} 55-2\end{array}$ \\
\hline Proxapertites tertiaria & $\mathrm{X}$ & & $\mathrm{X}$ & $\mathrm{X}$ & & & $\mathrm{X}$ & $\mathrm{x}$ & $\begin{array}{c}\text { 1-AS-37-AM } \\
577 / \mathrm{L} 65-4\end{array}$ \\
\hline Psilamonocolpites amazonicus & $\mathrm{X}$ & & & & & & $\mathrm{X}$ & & $\begin{array}{c}\text { 1-AS-37-AM } \\
579 / Y 55-4\end{array}$ \\
\hline Psilaperiporites multiporatus & & & $\mathrm{X}$ & & & & $\mathrm{X}$ & & $\begin{array}{c}\text { 1-AS-37-AM } \\
\text { 378/U65-1 }\end{array}$ \\
\hline Psilastephanoporites herngreenii & $\mathrm{X}$ & & $\mathrm{x}$ & & & $\mathrm{x}$ & $\mathrm{X}$ & & $\begin{array}{c}\text { 1-AS-37-AM } \\
557 / \mathrm{Y} 27\end{array}$ \\
\hline
\end{tabular}


Table 1. Cont.

\begin{tabular}{|c|c|c|c|c|c|c|c|c|c|}
\hline \multirow[b]{2}{*}{ Miospores } & \multicolumn{8}{|c|}{ Records in Solimões Formation* } & \multirow[b]{2}{*}{ No/EF } \\
\hline & 1-AS-04-AM & 1-AS-32-AM & $\begin{array}{l}\text { 1-AS-19-AM } \\
\text { 1-AS-27-AM }\end{array}$ & $\begin{array}{l}\text { 1-AS-51-AM } \\
\text { 1-AS-52-AM }\end{array}$ & $\begin{array}{l}\text { 1-AS-31-AM } \\
\text { 1-AS-34-AM }\end{array}$ & 1-AS-33-AM & 1-AS-105-AM & $\begin{array}{l}\text { Coari e Alto } \\
\text { Solimões }\end{array}$ & \\
\hline Psilastephanoporites tesseroporus & & & & $\mathrm{X}$ & & $\mathrm{X}$ & & $\mathrm{X}$ & $\begin{array}{c}\text { 1-AS-46-AM } \\
740 / \mathrm{H} 65-1\end{array}$ \\
\hline Psilatricolporites silvaticus & $\mathrm{X}$ & & & & & & $\mathrm{X}$ & & $\begin{array}{c}\text { 1-AS-46-AM } \\
755 / Y 52-3\end{array}$ \\
\hline Retistephanoporites crassinanulatus & $\mathrm{X}$ & & $\mathrm{X}$ & & & $\mathrm{X}$ & $\mathrm{X}$ & & $\begin{array}{c}\text { 1-AS-37-AM } \\
535 / \mathrm{T} 61\end{array}$ \\
\hline Retitrescolpites irregularis & $\mathrm{X}$ & & $\mathrm{X}$ & $\mathrm{X}$ & & $\mathrm{X}$ & $\mathrm{X}$ & $\mathrm{X}$ & $\begin{array}{c}\text { 1-AS-46-AM } \\
752 / Y 68-1\end{array}$ \\
\hline Retitrescolpites traversei & & & $\mathrm{X}$ & & & & $\mathrm{X}$ & & $\begin{array}{c}\text { 1-AS-37-AM } \\
422 / Y 41-4\end{array}$ \\
\hline Retitriporites dubiosus & $\mathrm{X}$ & & & $\mathrm{X}$ & & & & $\mathrm{X}$ & $\begin{array}{c}\text { 1-AS-37-AM } \\
533 / \mathrm{J} 61-1\end{array}$ \\
\hline Rhoipites guianensis & $\mathrm{X}$ & & $\mathrm{X}$ & $\mathrm{X}$ & & $\mathrm{X}$ & $\mathrm{X}$ & $\mathrm{X}$ & $\begin{array}{c}\text { 1-AS-46-AM } \\
755 / Y 51-2\end{array}$ \\
\hline Rhoipites toigoi & & & & & & & $\mathrm{x}$ & & $\begin{array}{c}\text { 1-AS-37-AM } \\
573 / \mathrm{M} 64-1\end{array}$ \\
\hline
\end{tabular}

* Based on the studies of Hoorn 1993 (1-AS-04-AM); Silva 2004 (1-AS-32-AM); Silva-Caminha et al. 2010 (1-AS-19-AM 1-AS-27-AM); Leandro 2012 (1-AS-51-AM 1-AS-52-AM); Kachniasz \& Silva-Caminha 2016 (1-AS-31-AM; 1-AS-34-AM); Leite et al. 2016 (1-AS-33AM); D’Apolito 2016 (1-AS-105-AM) and Silveira \& Souza 2015; 2016 (Coari e Alto Solimões outcrops).

Genus Kuylisporites Potonié 1956

Kuylisporites waterbolkii Potonié 1956 (Fig. 2G)

Botanical affinity: family Cyatheaceae, Cyathea horrida

Ecology: mountain areas (Jaramillo et al. 2010)

Genus Laevigatoporites Ibrahim

Laevigatosporites tibuiensis (Van der Hammen 1956a)

Jaramillo \& Dilcher 2001 (Fig. 2H)

Botanical affinity: unknown

Ecology: unknown

Genus Magnastriatites Germeraad et al. 1968, emend. Dettmann \& Clifford 1992

Magnastriatites grandiosus (Kedves \& Sole de Porta 1963) Dueñas 1980 (Fig. 2I)

Botanical affinity: Family Pteridaceae, genus

Ceratopteris

Ecology: aquatic genus (Tryon \& Lugardon 1991), rivers and shallow lakes (Jaramillo et al. 2010)

Genus Nijssenosporites

Nijssenosporites fossulatus Lorente 1986(Fig. 3A)

Botanical affinity: Family Adianthaceae, Genus

Pityrogramma

Ecology: pantropical genus (Tryon \& Lugardon 1991), plain.

Genus Perinomonoletes Krutzsch, 1967

Perinomonoletes sp. (Fig. 3B)

Botanical affinity: families Aspleniaceae/

Thelypteraceae (Jaramillo et al. 2010)

Ecology: unknown
Genus Polypodiaceoisporites Potonié 1951 ex Potonié 1956

Polypodiaceoisporites amazonensis Silva-Caminha et al. 2010 (Fig. 3C)

Botanical affinity: family Pteridaceae

Ecology: unknown

Polyapodiaceoisporites potoniei Kedves 1961 (Fig. 3D)

Botanical affinity: family Pteridaceae, Pteris

Ecology: open vegetation, riverine banks (Tryon \& Lugardon 1991).

Genus Polypodiisporites Potonié 1956 emend Khan and Martin 1971

Polypodiisporites aff. specious Sah 19671961 (Fig. 3E)

Botanical affinity: family Polypodiaceae

Ecology: plain (D’Apolito 2016)

Genus Psilatriletes van der Hammen 1954 ex Potonié 1956

Psilatriletes lobatus Hoorn 1994 (Fig. 3F)

Botanical affinity: unknown

Ecology: unknown

Genus Retitriletes Pierce 1961

Retitriletes sommeri Regali et al. 1974 (Fig. 3G)

Botanical affinity: family Lycopodiaceae?

Ecology: unknown

Genus Verrucatosporites Thomson \& Pflug 1953

Verrucatosporites usmensis (Van der Hammen 1956) 

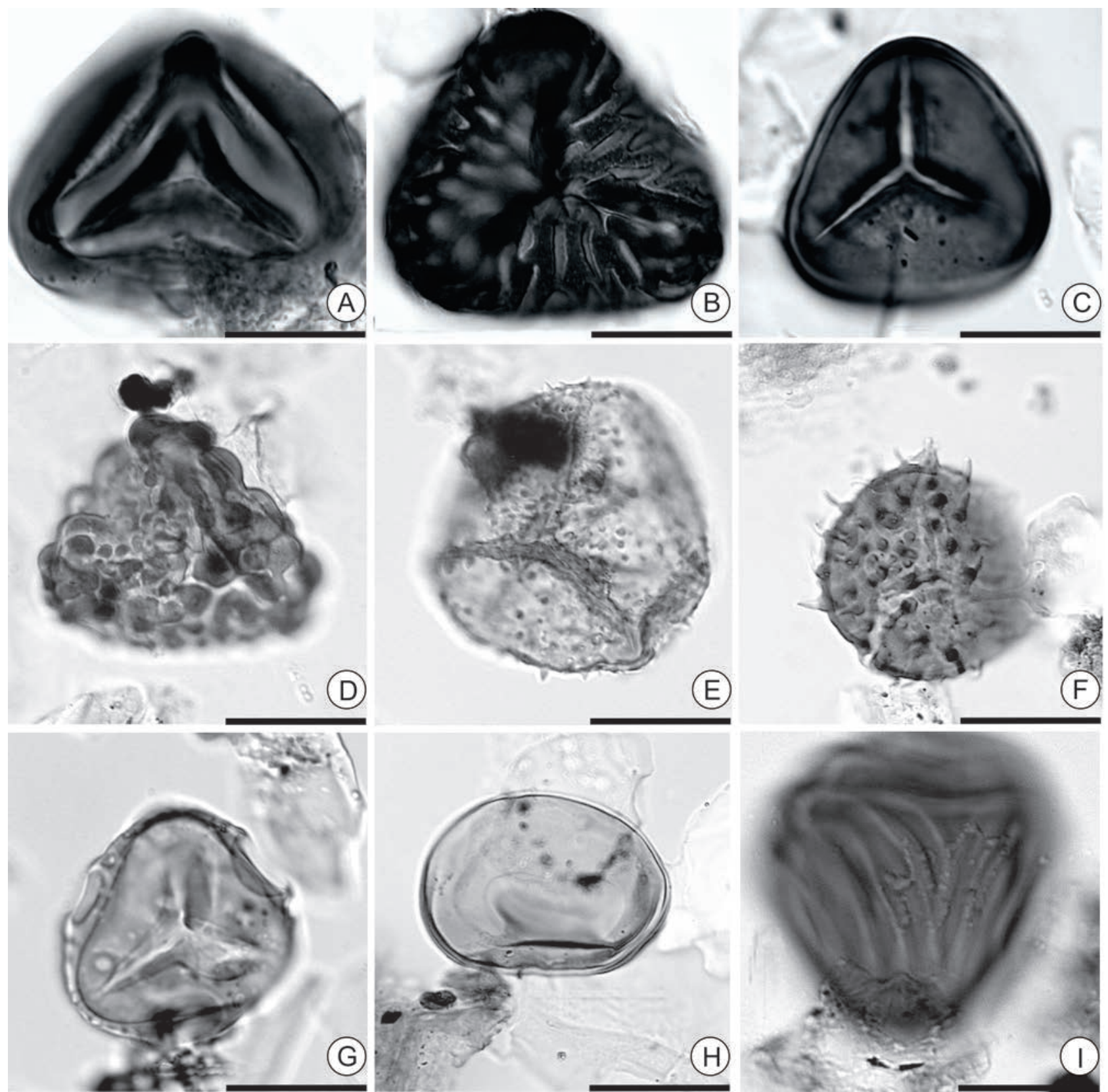

(E)
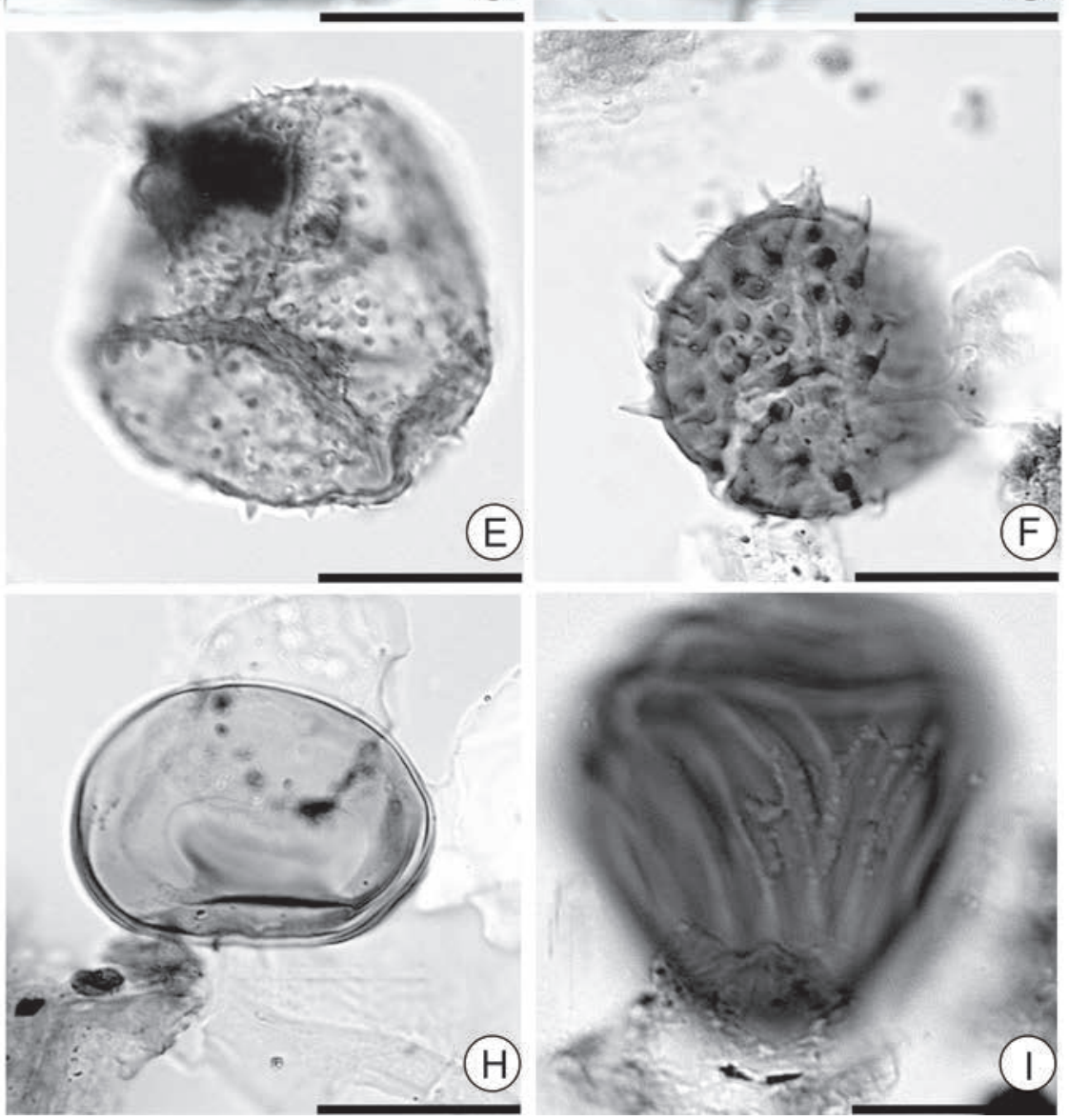

Figure 2. Fern spores recorded in the studied sections. A. Cingulatisporites laevigatus. B. Crassoretitriletes vanraadshoovenii. C. Deltoidospora adriennis. D. Distaverrusporites margaritatus. E. Echinatisporis infantus. F. Echinatisporis muelleri. G. Kuylisporites waterbolkii. H. Laevigatosporites tibuiensis. I. Magnastriatites grandiosus. Scale bar $=20 \mu \mathrm{m}$.

Germeraad et al. 1968 (Fig. 3H)

Botanical affinity: family Polypodiaceae, Stenochlaena palustris

Ecology: highland forest and e plain (Jaramillo et al. 2010)

Genus Verrucatotriletes Van Hoeken-Klinkenberg 1964

Verrucatotriletes bullatus Van Hoeken-Klinkenberg 1964 (Fig. 3I)

Botanical affinity: family Cyatheaceae, Alsophyla Ecology: pantropical genus (Tryon \& Lugardon1991), highland (Jaramillo et al 2010).

Verrucatotriletes etayoi Dueñas, 1980 (Fig. 3J)

Botanical affinity: unknown

Ecology: unknown

Anteturma POLLENITES Potonié 1893

Gimnosperms pollen grains

Genus Cyclusphaera Elsik 1966

Cyclusphaera scabrata Jaramillo \& Dilcher, 2001 (Fig. 4A)

Botanical affinity: Family Araucariaceae Ecology: mountain area 


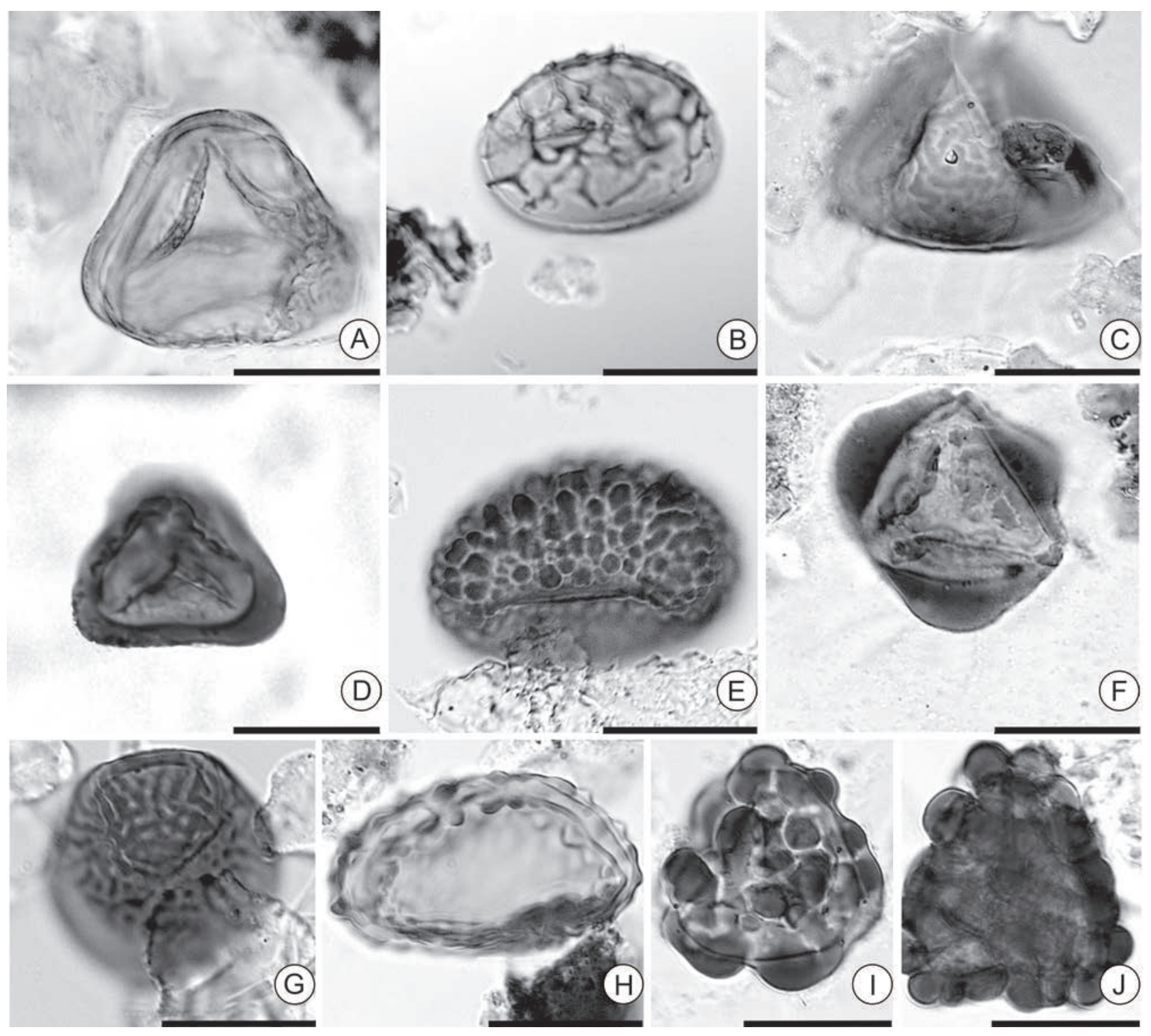

Figure 3. Fern spores recorded in the studied sections. A. Nijssenosporites fossulatus. B. Perinomonoletes. C. Polypodiaceoisporites amazonensis. D. Polypodiaceoisporites potoniei. E. Polypodiisporites aff. specious. F. Psilatriletes lobatus. G. Retitriletes sommeri. H. Verrucatoporites usmensis. I. Verrucatotriletes bullatus. J. Verrucatotriletes etayoi. Scale bar= $20 \mu \mathrm{m}$.

Genus Podocarpidites Cookson 1947

Podocarpidites sp. Cookson 1947 ex Couper 1953 (Fig. 4B)

Botanical affinity: family Podocarpaceae, Podocarpus Ecology: mountain and lownland forest (Jaramillo et al. 2010)

Angiosperms pollen grains

Genus Arecipites Wodehouse 1933, emend. Nichols et al. 1973

Arecipites perfectus Silva-Caminha et al 2010 (Fig. 4C)

Botanical affinity: family Arecaceae

Ecology: unknown

Genus Bombacacidites Couper 1960
Bombacacidites baculatus Muller et al. 1987 (Fig. 4D) Botanical affinity: family Bombacaceae, Pachira aquatica

Ecology: tropical forest, swamps and along rivers

Bombacacidites fossulatus Silva-Caminha et al. 2010

(Fig. 4E)

Botanical affinity: family Bombacaeae

Ecology: unknown

Bombacacidites lorenteae (Hoorn 1993) D’Apolito 2016 (Fig. 4F)

Botanical affinity: family Bombacaeae, Bombax

Ecology: along creeks and rivers 

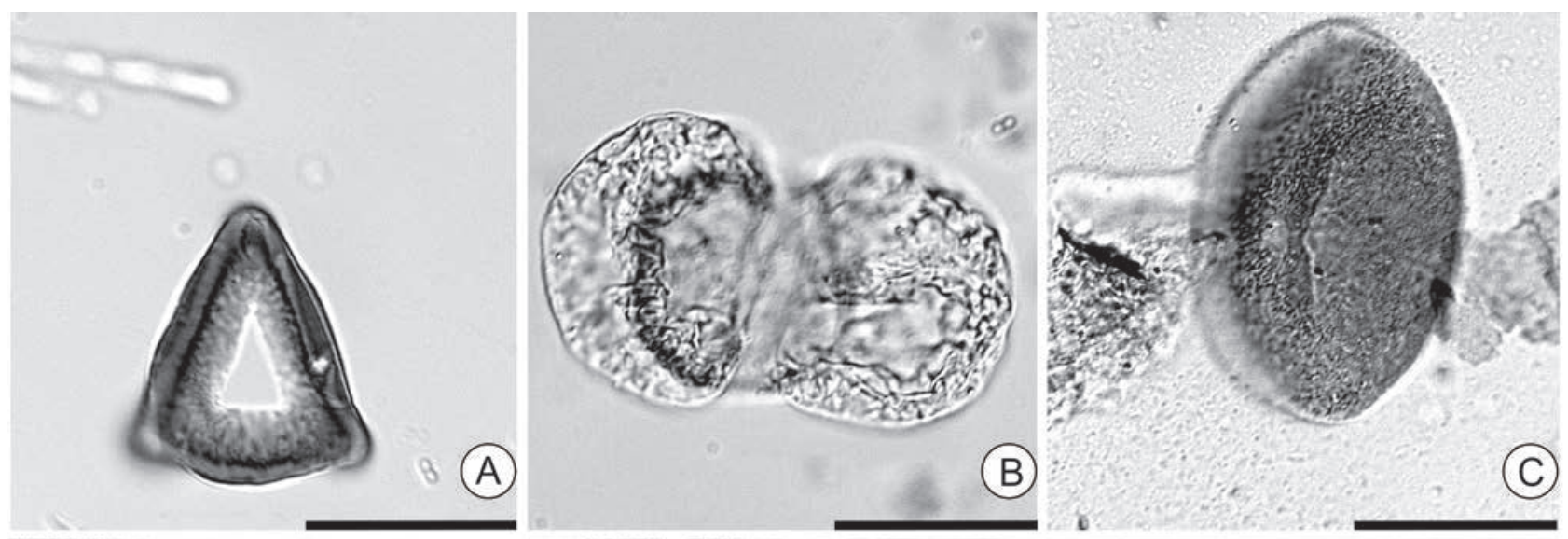

(B)
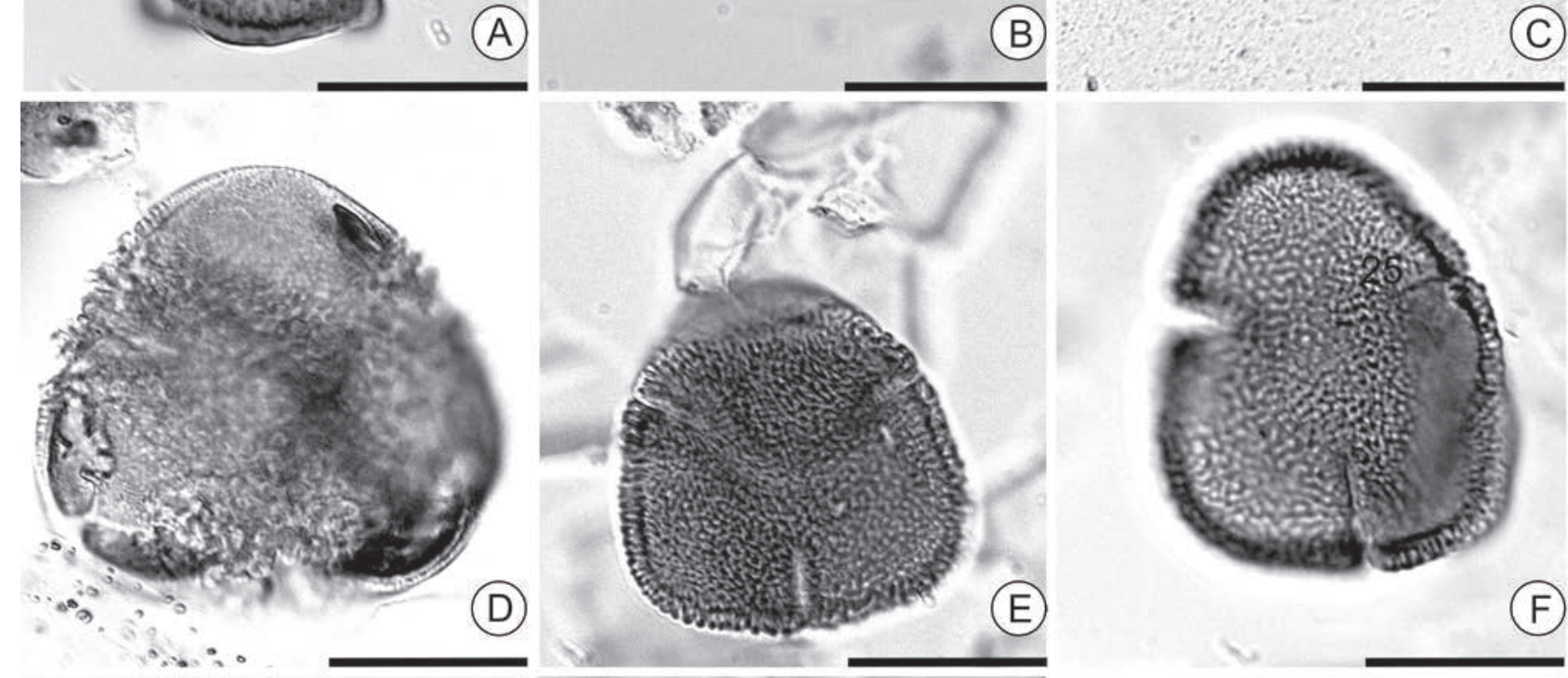

(C)
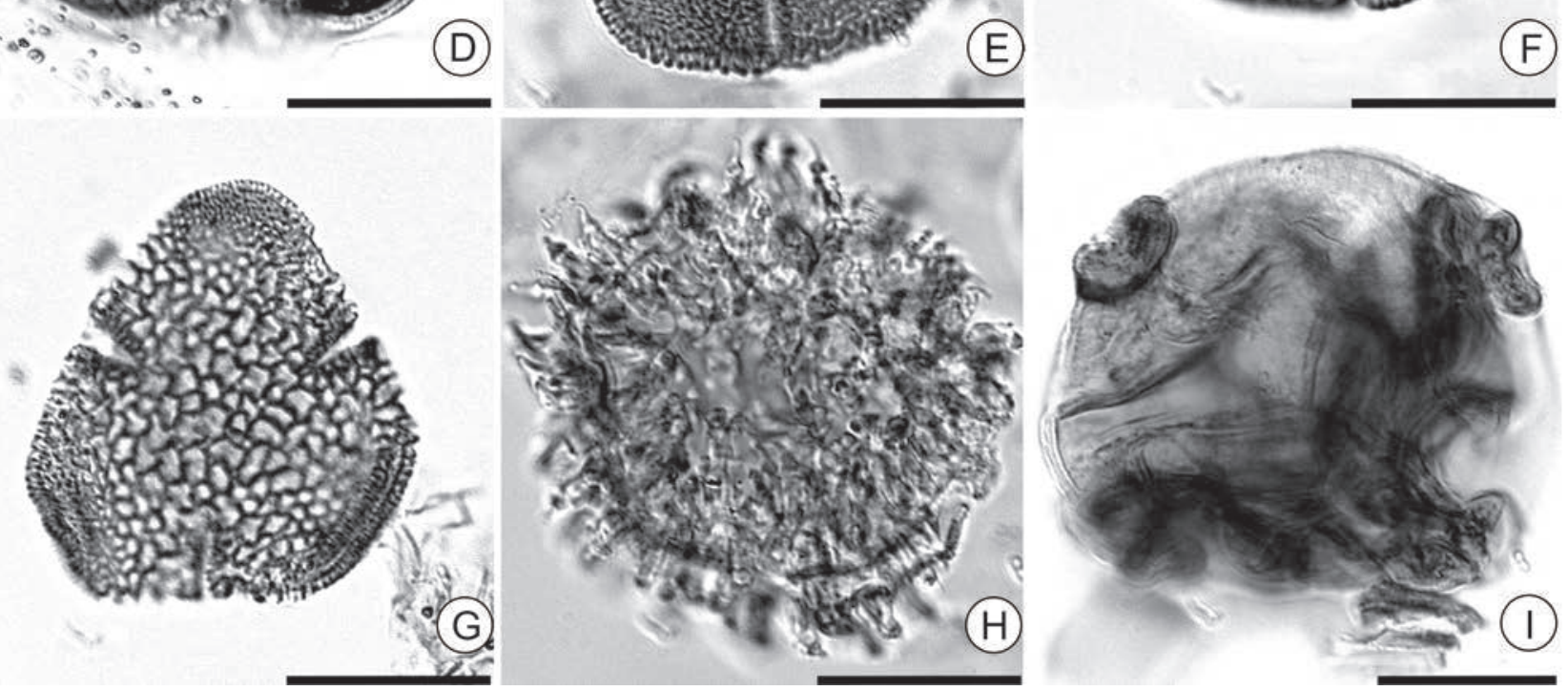

Figure 4. Pollen grains recorded in the studied sections. A. Cyclusphaera scabrata. B. Podocarpidites. C. Arecipites perfectus. D. Bombacacidites baculatus. E. Bombacacidites fossulatus. F. Bombacacidites lorenteae. G. Bombacacidites nacimientoensis. H. Cichoreacidites longispinosus. I. Corsinipollenites oculusnoctis (tetrad). Scale bar $=20 \mu \mathrm{m}$.

Bombacacidites nacimientoensis (Anderson1960) Elsik 1968 (Fig. 4G)

Botanical affinity: Family Bombacaeae, genus Bombax Ecology: plains, along watercourses (Jaramillo et al. 2010)

Genus Cichoreacidites Sah 1967

Cichoreacidites longispinosus (Lorente 1986) Silva-

Caminha 2010 (Fig. 4H)
Botanical affinity: family Asteraceae

Ecology: swamps, open vegetation and savannahs

Genus Corsinipollenites Nakoman 1965

Corsinipollenites oculusnoctis (tétrade) (Thiergart 1940); Nakoman (Fig. 4I)

Botanical affinity: family Onagraceae, Ludwigia Ecology: marshes (Jaramillo et al. 2010) 
Genus Echiperiporites Van der Hammem \& Wymstra 1964

Echiperiporites akanthos Van der Hammem \&

Wymstra 1964 (Fig. 5A)

Botanical affinity: family Alismataceae, Sagittaria/

Echinodorus

Ecology: swamps and lakes (D’Apolito 2016)

Echiperiporites estelae Germeraad et al. 1968 (Fig. 5B)

Botanical affinity: families Malvaceae/

Convolvulaceae

Ecology: coastal vegetation

Echiperiporites lophatus Silva-Caminha et al. 2010

(Fig. 5C)

Botanical affinity: family Convolvulaceae?

Ecology: herbaceous liana? (D’Apolito 2016)

Echiperiporites scabrannulatus Jaramillo et al. 2010

(Fig. 5D-E)

Botanical affinity: unknown

Ecology: unknown

Genus Echitricolporites Van der Hammen 1956 ex

Germeraad et al. 1968

Echitricolporites spinosus Van der Hammen 1956 ex

Germeraad et al. 1968 (Fig. 5F)

Botanical affinity: family Asteraceae

Ecology: open vegetation

Genus Echitriporites Van der Hammen 1956

Echitriporites trianguliformis Van Hoeken Klinkenberg 1964 (Fig. 5G)

Botanical affinity: family Proteaceae (Jaramillo \&

Rueda 2013)

Ecology: unknown

Genus Fenestrites Van der Hammen, 1956

Fenestrites garciae Leite 2006 (Fig. 5H)

Botanical affinity: family Amaranthaceae, Gomphrena Ecology: unknown

Fenestrites spinosus Van der Hammen, 1956 (Fig. 5I) Botanical affinity: family Asteraceae (Germeraad et al. 1968)

Ecology: unknown

Genus Grimsdalea Germeraad et al. 1968

Grimsdalea magnaclavata Germeraad et al. 1968 (Fig.

5J)

Botanical affinity: family Arecaceae?

Ecology: unknown

Genus Inaperturopollenites Nilsson 1958

Inaperturopollenites solimoensis Leite 2006 (Fig. 5K)

Botanical affinity: family Rubiaceae, Psychotria?

Ecology: unknown
Genus Ladakhipollenites Mathur \& Jain 1980

Ladakhipollenites? caribbiensis (Muller et al. 1987)

Silva-Caminha et al. 2010 (Fig. 5L)

Botanical affinity: family Euphorbiaceae, Sapium

Ecology: terra firme forest and varzea forest

Genus Loranthacites Mtchedlishvili in Samoilovitch \& Mtchedlishvili 1961

Loranthacites digitatus Silva-Caminha et al. 2010

(Fig. 6A)

Botanical affinity: family Loranthaceae (Jaramillo \&

Rueda 2013)

Ecology: unknown

Genus Malvacipollis Harris 1965

Malvacipollis spinulosa Frederiksen, 1983 (Fig. 6B-C)

Botanical affinity: family Euphorbiaceae (Leite 2006)

Ecology: unknown

Genus Malvacipolloides Anzótegui \& Garalla 1986

Malvacipolloides maristellae (Muller et al. 1987) Silva-

Caminha et al 2010 (Fig. 6D)

Botanical affinity: family Bombacaceae

Ecology: terra firme forest

Genus Margocolporites Ramanujam 1966 ex

Srivastava 1969, emend. Pocknall \& Mildenhall 1984

Margocolporites "hornii" (Fig. 6E)

Botanical affinity: family Apocynaceae?

Ecology: unknown

Margocolporites vanwijhei Germeraad et al. 1968 (Fig.

6F)

Botanical affinity: family Caesalpiniaceae, Caesalpinea Ecology: coastal vegetation

Genus Mauritiidites van Hoeken-Klinkenberg 1964

Mauritiidites franciscoi var. franciscoi Van der

Hammen, 1956) Van Hoeken Klinkenberg 1964 (Fig.

6G)

Botanical affinity: family Arecaceae, Mauritia

Ecology: plain and swamps

Genus Perisyncolporites Germeraad et al. 1968

Perisyncolporites pokornyi Germeraad et al. 1968 (Fig.

6H)

Botanical affinity: family Malpighiaceae

Ecology: plain and terra firme forest

Genus Polyadopollenites Pflug and Thomson 1953

Polyadopollenites marileae Leite 2006 (Fig. 6I)

Botanical affinity: family Mimosaceae

Ecology: unknown

Genus Proteacidites Cookson emend. Couper 1953

Proteacidites triangulatus Lorente 1986 (Fig. 6J) 

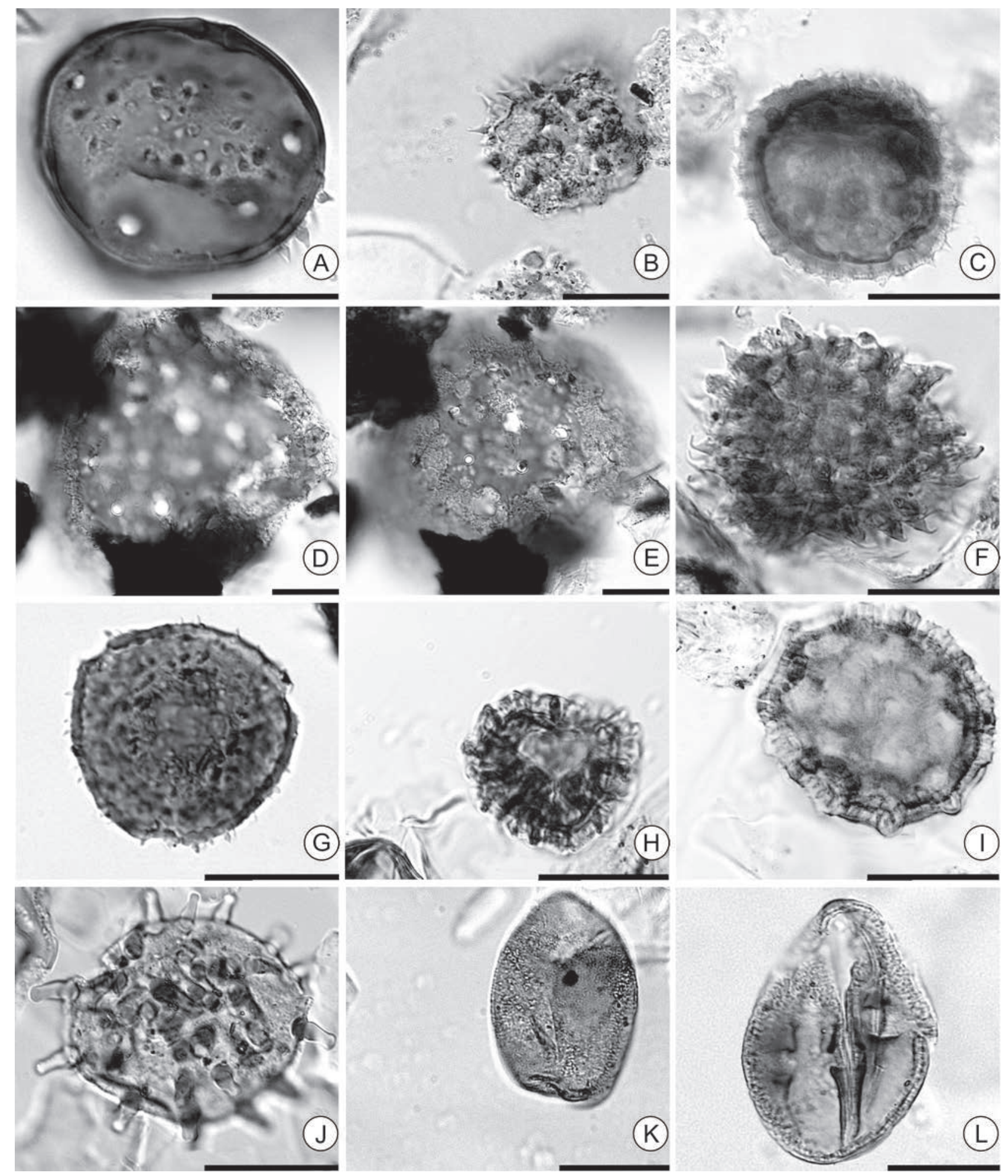

Figure 5. Pollen grains recorded in the studied sections. A. Echiperiporites akanthos. B. Echiperiporites estelae. C. Echiperiporites lophatus. D-E. Echiperiporites scrabrannulatus. F. Echitricolporites spinosus. G. Echitriporites trianguliformis. H. Fenestristes garciae. I. Fenestrites spinosus. J. Grimsdalea magnaclavata. K. Inaperturopollenites solimoensis. L. Ladakhipollenites? caribbiensis. Scale bar $=20 \mu \mathrm{m}$. 

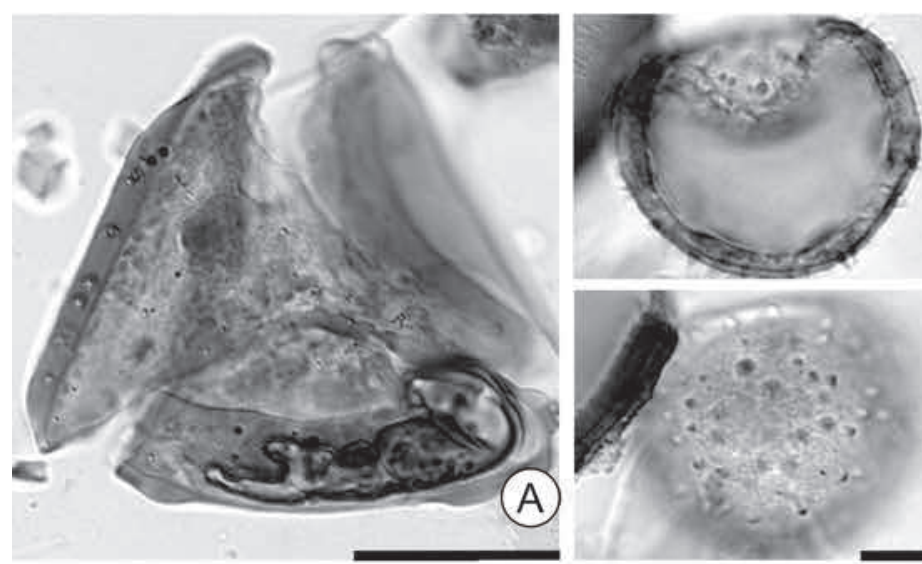

(B)

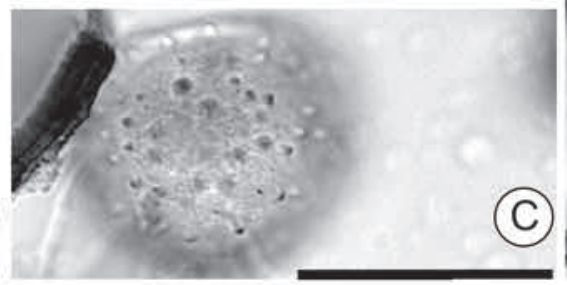

(C)
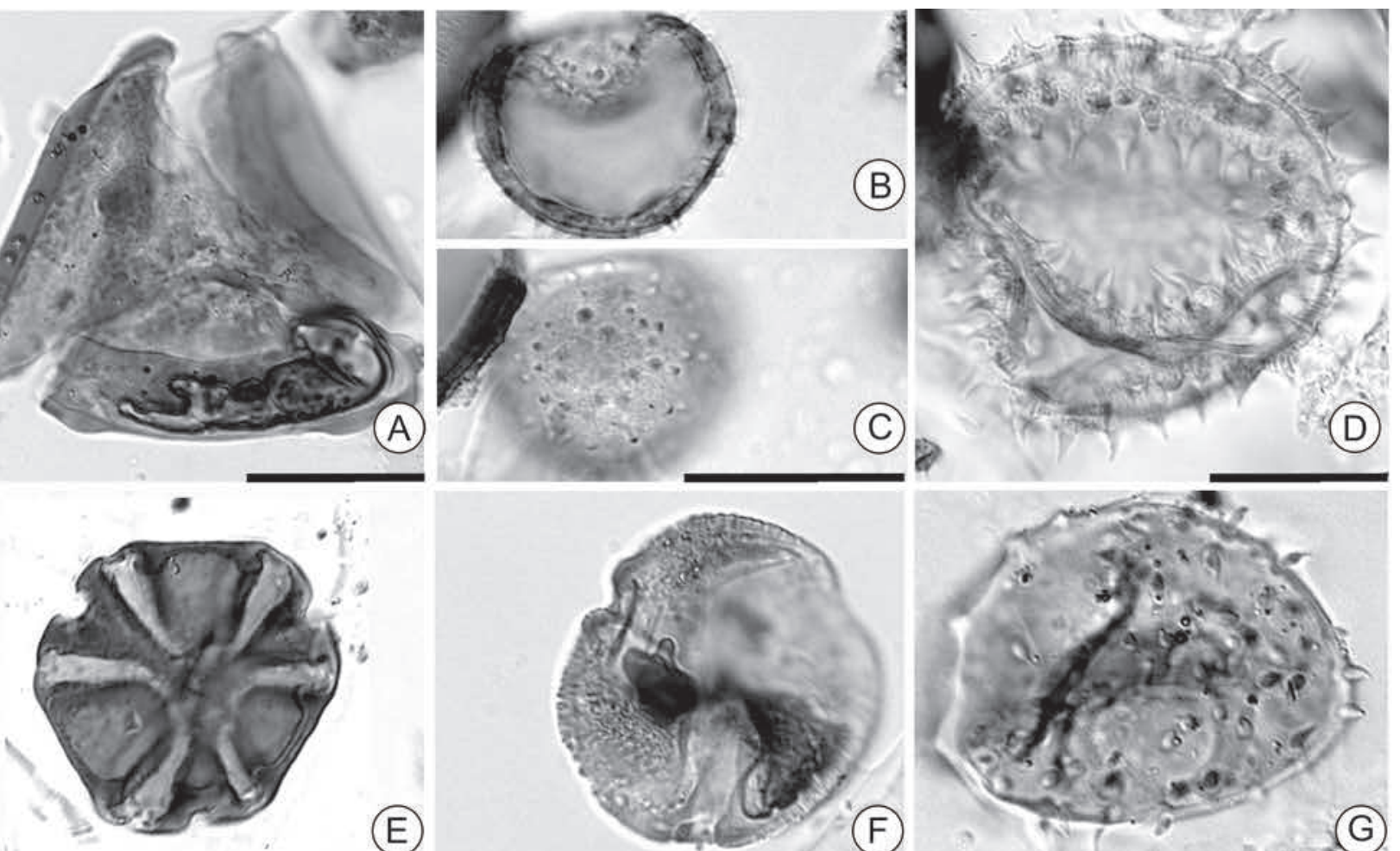

(E)
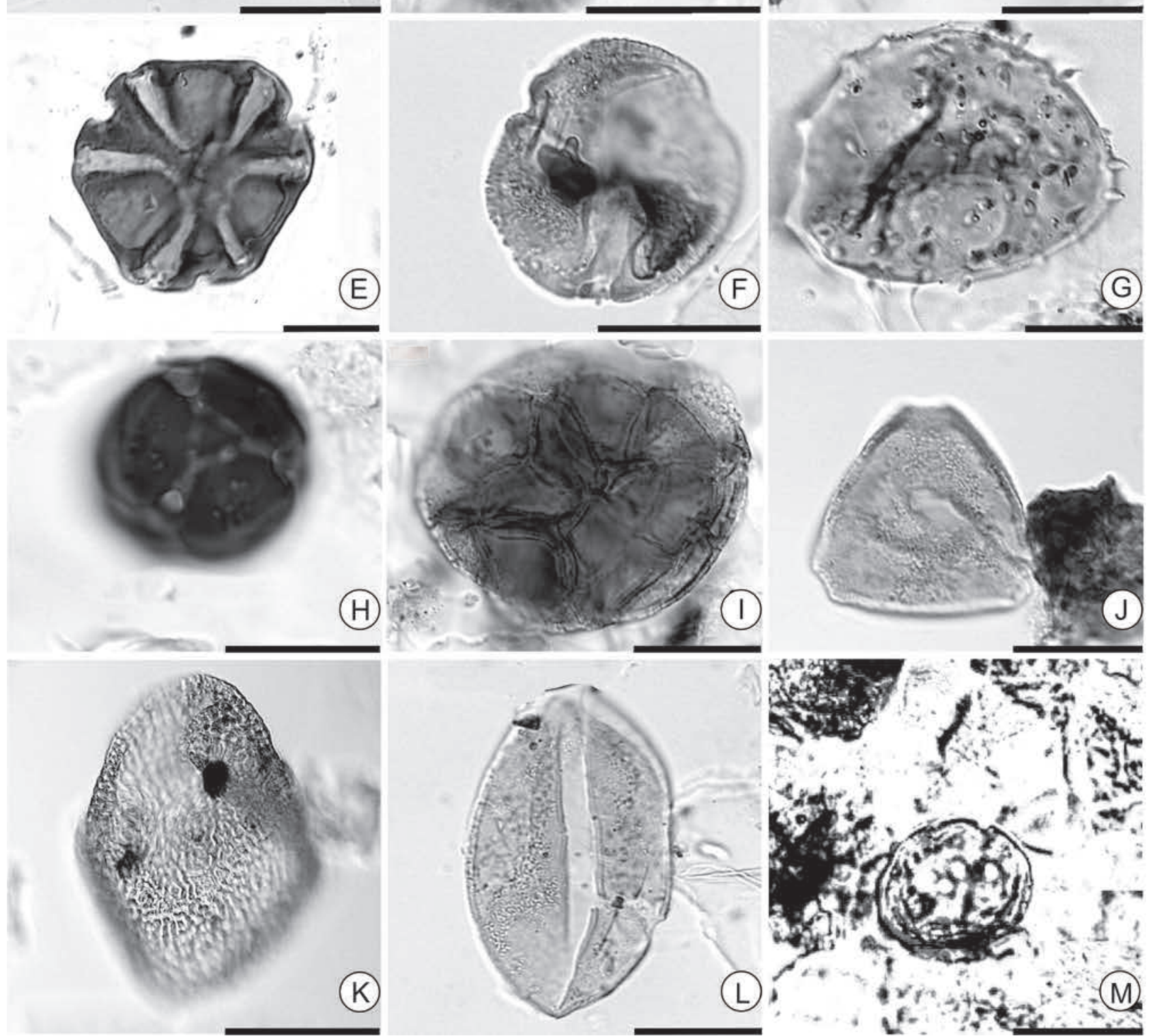

Figure 6. Pollen grains recorded in the studied sections. A. Loranthacites digitatus. B-C. Malvacipollis spinulosa. D. Malvacipolloides maristellae. E. Margocolporites "hornii". F. Margocolporites vanwijhei. G. Mauritiidites franciscoi var. franciscoi. H. Perisyncolporites pokornyi. I. Polyadopollenites marileae. J. Proteacidites triangulatus. K. Proxapertites tertiaria. L. Psilamonocolpites amazonicus. M. Psilaperiporites multiporatus. Scale bar $=20 \mu \mathrm{m}$. 
Botanical affinity: families Sapindaceae/Proteaeceae Ecology: tropical forest and montane forest

Genus Proxapertites Van der Hammen 1956

Proxapertites tertiaria Van der Hammen \& García de Mutis 1966 (Fig. 6K)

Botanical affinity: family Annonaceae, Crematosperma Ecology: lowland forest (Jaramillo et al. 2010).

Genus Psilamonocolpites Van der Hammen \& C. Garcia de Mutis 1966

Psilamonocolpites amazonicus Hoorn 1993 (Fig. 6L)

Botanical affinity: family Arecaceae, Euterpe

Ecology: varzea forest, plain and swamps

Genus Psilaperiporites Regali et al. 1974

Psilaperiporites multiporatus Hoorn 1994 (Fig. 6M)

Botanical affinity: unknown

Ecology: unknown

Genus Psilastephanoporites Van der Hammen 1956

Psilastephanoporites herngreenii Hoorn 1993 (Fig. 7A)

Botanical affinity: family Apocynaceae

Ecology: lowland forest

Psilastephanoporites tesseroporus Regali et al. 1974

(Fig. 7B)

Botanical affinity: family Apocynaceae, Prestonia?

(Leite 2006)

Ecology: unknown

Genus Psilatricolporites Pierce 1961

Psilatricolporites silvaticus Hoorn 1993 (Fig. 7C)

Botanical affinity: family Burseraceae/Sapotaceae

Ecology: lowland forest

Genus Retistephanoporites González-Guzmán 1967

Retistephanoporites crassinanulatus Lorente 1986 (Fig.

7D)

Botanical affinity: family Malvaceae, Quararibea

Ecology: lowland forest

Genus Retitrescolpites Sah 1967

Retitrescolpites irregulares (Van der Hammen \&

Wymstra 1964) Jaramillo \& Dilcher 2001 (Fig. 7E)

Botanical affinity: family Euphorbiaceae, Amanoa

Ecology: lowland forest, along watercourses

(Jaramillo \& Rueda 2013)

Retitrescolpites traversei Silva-Caminha et al 2010 (Fig. 7F)

Botanical affinity: family Acanthaceae, Teliostachya

Ecology: lowland forest

Genus Retitriporites (Van der Hammen, 1956)

Gonzalez-Guzmán, 1967
Retitriporites dubiosus Gonzalez-Guzmán, 1967 (Fig.

7G)

Botanical affinity: family Rubiaceae, Psychotria/

Alibertia (Leite 2006)

Ecology: unknown

Genus Rhoipites Wodehouse 1933

Rhoipites guianensis (Van der Hammen \& Wymstra

1964) Jaramillo \& Dilcher 2001(Fig. 7H-I)

Botanical affinity: family Malvaceae, Firmiana/

Hildegardia/Glossostemon/ Trichospermum (Germeraad

et al 1968)

Ecology: unknown

Rhoipites toigoi D’Apolito 2016 (Fig. 7J)

Botanical affinity: family Rubiaceae?

Ecology: unknown

\section{Discussion}

This catalogue lists 60 taxa miospores present in the Miocene of the Brazilian Amazon, with photomicrographs and brief description of botanical affinity, ecology and distribution. This serves to support for paleoenvironmental and biostratigraphic studies for the region.

The assemblage of miospores is well preserved and well diversified. The spores are arranged in 16 genera and 19 species, included in ten families, with Pteridaceae the most representative family followed by Polypodiaceae and Cyatheaceae.

In Solimões Formation, the fern spores Crassoretitriletes vanhadshoovenii Magnastriatites grandiosus and Deltoidospora adriennis were recorded in almost all those previous studies. These species have habitats related to the aquatic environment as mangroves, swamps, rivers and lakes.

The spermatophytes group (pollen grains) encompasses two families of gymnosperms: Aracauariaceae (Cyclusphaera scabrata) and Podocarpaceaeae (Podocarpidites). It is the second record of the family Araucariaceae of the Solimões Formation. And the angiosperms are represented by 20 families, containing 31 genera and 41 species. The genus Bombacacidites and Echiperiporites exhibit the largest number of species.

The angiosperms species with numerous records in Solimões Formation were Bombacacidites baculatus, Corsinipollenites oculusnoctis, Echiperiporites estelae, Echitricolporites spinosus, Grimsdalea magnaclavata, Mauritiidites franciscoi and Perisyncolporites pokornyi, which indicate water-related environments such as plains, mangroves, marshes and coast vegetation, except E. spinosus, typical of open vegetation.

Two new miospores were recorded for Solimões Formation: the fern spore Retitriletes sommeri and the pollen grain Echiperiporites scabrannulatus. 


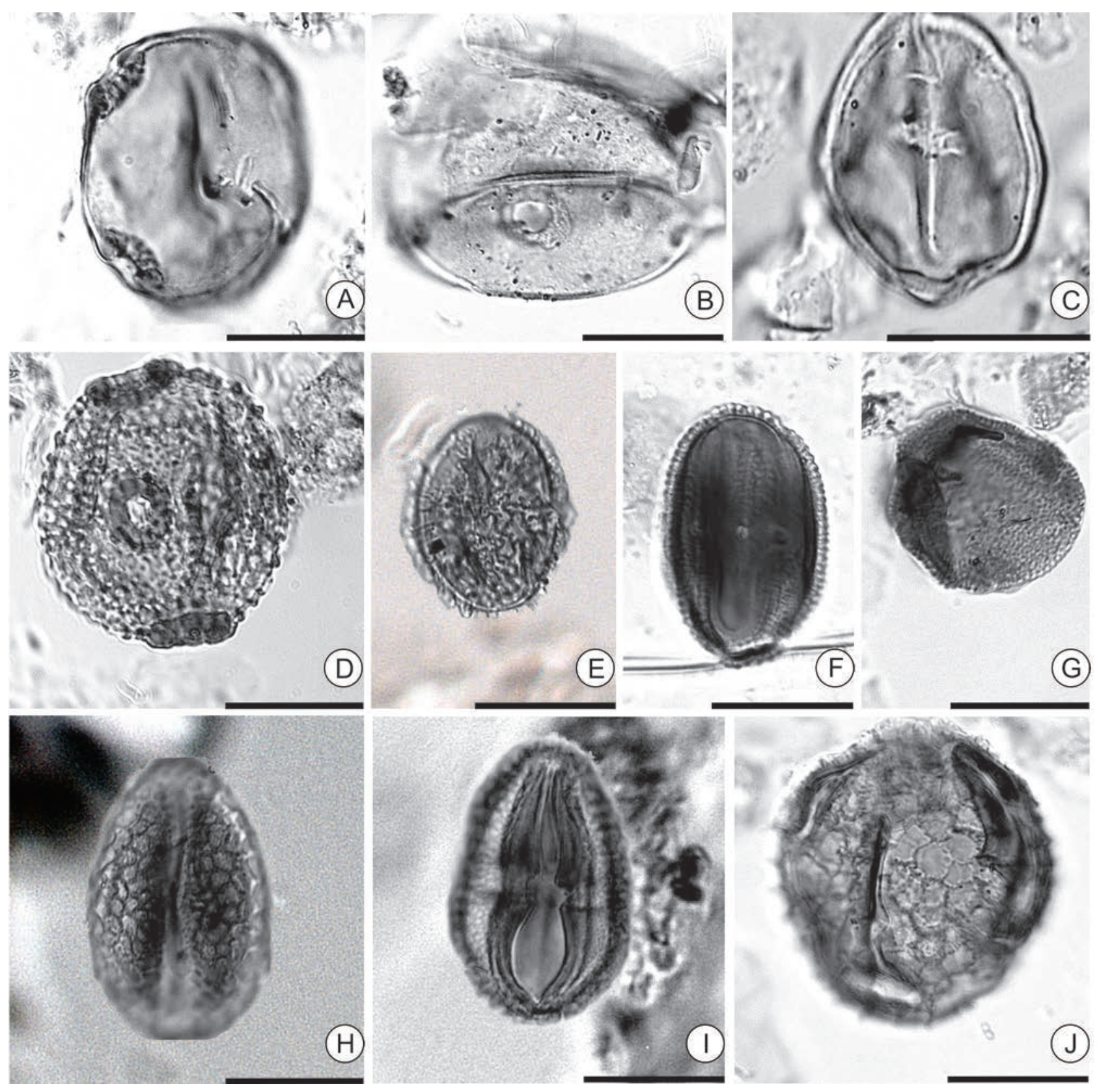

Figure 7. Pollen grains recorded in the studied sections. A. Psilastephanoporites herngreenii. B. Psilastephanoporites tesseroporus. C. Psilatricolporites silvaticus. D. Retistephanoporites crassinanulatus. E. Retitrescolpites irregularis. F. Retitrescolpites traversei. G. Retitriporites dubiosus. H-I. Rhoipites guianensis. J. Rhoipites toigoi. Scale bar $=20 \mu \mathrm{m}$.

\section{Acknowledgements}

We express our thanks to the Geological Survey of Brazil (CPRM) for giving N.P. Sá the opportunity to study the material and the Brazilian National Council for Scientific and Technological Development (Conselho Nacional de Desenvolvimento Científico e Tecnológico - CNPq) for the scholarship and the financial support to N.P. Sá (Grant no. 140408/2013-4). We thank three anonymous reviewers for helpful suggestions.

\section{References}

Barata CF, Caputo MV. 2007. Geologia do petróleo da Bacia do Solimões - O Estado da Arte. Campinas, PDPETRO.

Boonstra M, Ramos MIF, Lammertsma EI, Antonie PO, Hoorn C. 2015. Marine connections of Amazonia: Evidence from foraminífera and dinoflagellate cysts (early to middle Miocene, Colombia/Peru). Palaeogeography, Palaeoclimatology, Palaeoecology 417: 176-194.

Burnham RJ, Graham A. 1999. The history of Neotropical vegetation: new developments and status. Annais Missouri Botanical Garden 86: 546-589. 
Cozzuol M. 2006. The Acre vertebrate fauna: diversity, geography and time. Journal of South American Earth Sciences 21: 185-203.

Cronquist A. 1988. The evolution and classification of flowering plants. 2nd. edn. New York, New York Botanical Garden.

D'Apolito C. 2016. Landscape evolution in Western Amazonia: palynostratigraphy, palaeoenvironments and diversity of the Miocene Solimões formation, Brazil. PhD Thesis, University of Birmingham, Birmingham.

Eiras JF, Becker CR, Souza EM, Gonzaga FG, Silva JGF, Daniel LMF. 1994 Bacia do Solimões. Boletim de Geociências da Petrobrás 8: 17-45.

Erdtman G. 1969. Handbook of palynology. Copenhagen, Scandinavian University Books.

Faegri K, Iversen J. 1966. Textbook of pollen analysis. Copenhagen, Scandinavian University Books.

Frailey CD. 1986. Late Miocene and Holocene mammals, exclusive of the Notoungulata, of the Rio Acre region, Western Amazonia. Contribution in Science, Natural History Museum of Los Angeles County 374: 1-46.

Gentry AH. 1982. Neotropical floristic diversity: phytogeographical connections between Central and South America, Pleistocene climatic fluctuations, or an accident of the andean orogeny? Annals of the Missouri Botanical Garden 69: 557-593.

Germeraad JH, Hopping CA, Muller J. 1968. Palynology of Tertiary sediments from tropical areas. Review of Paleobotany and Palynology 6: 189-348.

Gross M, Piller WE, Ramos MI, Paz JDS. 2011. Late Miocene sedimentary environments in south-western Amazonia (Solimões Formation; Brasil). Journal of South American Earth Sciences 32: 169-181.

Gross M, Ramos MI, Caporaletti M, Pilner WE. 2013. Ostracods (Crustacea) and their palaeoenviromental implication for the Solimões Formation (Late Miocene; Western Amazonia/Brazil). Journal of South American Earth Sciences 42: 216-241.

Haffer J. 1969. Speciation in amazonian forest birds. Science 165: 131-137.

Hooghiemstra H, Hammen T. 1998. Neogene and Quaternary development of the Neotropical rain forest: the forest refugia hypothesis, and a literature overview. Earth - Science Review 44: 147-183.

Hoorn C. 1993. Marine incursions and the influence of Andean tectonics on the Miocene depositional history of northwestern Amazônia: results of Palynostratigraphic study. Palaegeography, Palaeoclimatology, Palaeocology 105: 267-309.

Hoorn C. 1994a. Fluvial palaeoenvironments in intracratonic Amazonas Basin (Early Miocene-Early Middle Miocene, Colombia). Palaegeography, Palaeoclimatology, Palaeocology 109: 1-54.

Hoorn C. 1994b. An environmental reconstruction of the palaeo-Amazon River sytem (Middle to Late Miocene, NW Amazonia). Palaeogeography, Palaeoclimatology, Paleoecology 112: 187-238.

Hoorn C. 1994c. Miocene palynostratigraphy and paleoenvironments of Northwestern Amazonia. PhD Thesis, University of Amsterdan, Amsterdan.

Hoorn C, Bernardes-de-Oliveira MEC, Dino R, et al. 2010c. Neogene climate evolution in Amazonia and the Brazilian Northeast. In: Carvalho IS, Garcia MJ, Lana CC, Strohschoen Jr O. (eds.) Paleontologia: Cenários de Vida-Paleoclimas. Rio de Janeiro, Interciência. p. 277-310.

Hoorn C, Bogotá-A GR, Romero-Baez M, et al. 2017. The Amazon at sea: Onset and stages of the Amazon River from a marine record, with special reference to Neogene plant turnover in the drainage basin. Global and Planetary Change 153: 51-65.

Hoorn C, Guerreiro J, Sarmiento G. 1995. Andean tectonics as a cause for changing drainage patterns in Miocene Northern South America. Geology 23: 237-240.

Hoorn C, Wesselingh FP, Hovikoski J, Guerrero J. 2010b. The Amazonian mega-wetland (Miocene; Brazil, Colombia, Peru, Bolivia). In: Hoorn C, Wesselingh FP. (eds.) Amazonia, Landscape and Species Evolution: Look into the Past. Oxford, Wiley-Blackwell. p. 123-143.

Hoorn C, Wesselingh FP, Steege H, et al. 2010a. Amazonia Through Time: Andean uplift, climate change, landscape evolution, and biodiversity. Science 330: 927-931.

Jaramillo C, Hoorn C, Silva SAF, et al. 2010. The origin of the modern Amazon rainforest: implications of the palynological and paleobotanical record. In: Hoorn C, Wesselingh FP. (eds.) Amazonia: landscape and species evolution - a look into the past. Oxford, Blackwell Publishing. p. 317-334

Jaramillo C, Rueda MJ. 2013. A morphological electronic database of Cretaceous Tertiary and extant pollen and spores from Northern South America. v. 2012/2013. http://biogeodb.stri.si.edu/jaramillo/ palynomorph/. 25 Apr. 2017.

Jaramillo C, Rueda M, Mora G. 2006. Cenozoic plant diversity in the Neotropics. Science 311: 1893-1896.

Jaramillo C, Rueda M, Torres V. 2011. A palynological zonation for the Cenozoic of the Llanos and Llanos Foothills of Colombia. Palynology 35: 46-84.

Kachniasz KE, Silva-Caminha SAF. 2016. Palinoestratigrafia da Formação Solimões: comparação entre bioestratigrafia tradicional e o método de associações unitárias. Revista Brasileira de Paleontologia 19: 481-490.

Latrubesse E, Bocquentin J, Santos JCR, Ramonell CG. 1997. Paleoenvironmental model for the late Cenozoic southwestern Amazonia paleontology and geology. Acta Amazonica 27: 103-118.

Latrubesse E, Caminha-Silva S, Cozzuol M, Absy M. 2007. Late Miocene continental sedimentation in the southwestern Amazonia and its regional significance: Biotic and geological evidence. Journal of South American Earth Science 23: 61-80.

Latrubesse E, Cozzuol M, Rigsby C, Silva S, Absy ML, Jaramillo C. 2010. The Late Miocene paleogeography of the Amazon Basin and the evolution of the Amazon River system. Earth-Science Reviews 99: 99-124.

Leandro LM. 2012. Análises palinoestratigráficas da Formação Solimões na porção noroeste do Alto Solimões, Amazonas, Brasil. MSc Thesis, Universidade do Vale do Rio dos Sinos, São Leopoldo.

Leite FPR. 2006. Palinologia da Formação Solimões, Neógeno da Bacia do Solimões, estado do Amazonas: Implicação paleoambientais e bioestratigráficas. PhD Thesis, Universidade de Brasília, Brasília.

Leite FPR, Paz J, Carmo DA, Silva-Caminha S. 2016. The effects of the inception of Amazonian transcontinental drainage during the Neogene on the landscape and vegetation of the Solimões Basin, Brazil. Palynology 40: 1-11.

Linhares AP, Ramos MIF, Gross M, Piller WE. 2011. Evidence for marine influx during the Miocene in southwestern Amazonia, Brazil. Geología Colombiana 36: 91-104.

Lorente M. 1986. Palynology and palynofacies of the Upper Tertiary in Venezuela. Cramer, Berlin/Stuttgart Band, Dissertationes Botanicae.

Lovejoy N. 1998. Marine incursion into South America. Nature 396: 421-422.

Maia RGN, Godoy HK, Yamaguti HS, et al. 1977. Projeto de carvão no Alto Solimões. Relatório Final. Manaus, CPRM-DNPM.

Monsch KA. 1998. Miocene fish faunas from the northwestern Amazonia basin (Colombia, Peru, Brazil) with evidence of marine incursions. Palaeogeography, Palaeoclimatology, Palaeoecology 143: 31-50.

Nogueira ACR, Silveira R, Guimarães JTF. 2013. Neogenee-Quaternary sedimentary and paleovegetation history of the eastern Solimões Basin, central Amazon region. Journal of South American Earth Sciences 46: 89-99.

Ramos MI. 2006. Ostracods from the Neogene Solimões Formation (Amazonas, Brazil). Journal of South American Earth Sciences 21: 87-95.

Räsänen ME, Linna AM, Santos JCR, Negri FR. 1995. Late Miocene tidal deposits in the Amazonian foreland basin. Science 269: 386-389.

Regali MSP, Uesugui N, Santos AS. 1974a. Palinologia dos sedimentos mesocenozóicos do Brasil - I. Boletim Técnico da Petrobrás 17: 177-191.

Regali MSP, Uesugui N, Santos AS. 1974b. Palinologia dos sedimentos mesocenozóicos do Brasil - II. Boletim Técnico da Petrobrás 17: 263-301.

Silva, SAF. 2004. Palinologia do Neógeno da Amazônia Sul Ocidental, Brasil. MSc Thesis, Instituto Nacional de Pesquisas da Amazônia, Manaus.

Silva-Caminha SAF, Jaramillo CA, Absy ML. 2010. Neogene palynology of the Solimões Basin, Brazilian Amazonia. Palaeontographica 283: 1-67.

Silveira RR, Souza PA. 2015. Palinologia (grãos de pólen de angiospermas) das formações Solimões e Içá (bacia do Solimões), nas regiões de Coari e Alto Solimões, Amazonas. Revista Brasileira de Paleontologia, 18: 455-474.

Silveira RR, Souza PA. 2016. Palinologia (esporos de fungo e pteridófitas, grãos de pólen de gimnospermas, cistos de algas e escolecodonte) das 
formações Solimões e Içá (Neógeno e Pleistoceno, Bacia do Solimões), Amazonas, Brasil. Pesquisas em Geociências 43:17-39.

Tryon AF, Lugardon B. 1991. Spores of the Pteridophyta. Surface, wall structure and diversity based on electron microscope studies. New York, Springer.

Uesugui N. 1979. Palinologia; técnicas de tratamento de amostras. Boletim Técnico da Petrobrás 22: 229-240.

Vonhof HB, Wesselingh FP, Ganssen GM. 1998. Reconstruction of the Miocene western Amazonian aquatic system using molluscan isotopic signatures. Palaeogeography, Palaeoclimatology, Paleoecology 141: 85-93.

Vonhof HB, Wesselingh FP, Kaandorp RJG. 2003. Paleogeography of Miocene western Amazonia: isotopic composition of molluscan shells constrains the influence of marine incursions. Geology Society of America Bulletin 115: 983-993.

Wanderley-Filho JR, Eiras JF, Vaz PT, et al 2007. Bacia do Solimões. Boletim de Geociências da Petrobrás 15: 217-225.

Wesselingh FP. 2006. Molluscs from the Miocene Pebas Formation of Peruvian and Colombian Amazonia. Scripta Geologica 133: 19-290. Wesselingh FP, Ranzi A, Räsänen ME. 2006. Miocene freshwater Mollusca from western Brazilian Amazonia. Scripta Geologica 133: 419-437. Wesselingh FP, Räsänen ME, Irion G, et al. 2002. Lake Pebas: a palaeoecological reconstruction of a Miocene, a long-lived lake complex in western Amazonia. Cainozoic Research 1: 35-81.

Wesselingh FP, Salo JA. 2006. A Miocene perspective on the evolution of the Amazonian biota. Scripta Geologica 133: 439-458. 